\title{
Multivariate Likelihood Ratio Order for Skew-Symmetric Distributions with a Common Kernel
}

\author{
Werner Hürlimann \\ Wolters Kluwer Financial Services, Seefeldstraße 69, 8008 Zürich, Switzerland \\ Correspondence should be addressed to Werner Hürlimann; whurlimann@bluewin.ch
}

Received 2 October 2013; Accepted 24 October 2013

Academic Editors: A. Hutt, E. Marchand, C. A. Tudor, and A. Volodin

Copyright (C) 2013 Werner Hürlimann. This is an open access article distributed under the Creative Commons Attribution License, which permits unrestricted use, distribution, and reproduction in any medium, provided the original work is properly cited.

The multivariate likelihood ratio order comparison of skew-symmetric distributions with a common kernel is considered. Two multivariate likelihood ratio perturbation invariance properties are derived.

\section{Introduction}

According to Azzalini and Capitanio [1], the density function of the multivariate skew-symmetric distribution (SSD) with centrally symmetric (about 0 ) density kernel $f_{0}(x)$, absolutely continuous univariate skewing distribution $G_{0}(x)$ with an even density $g_{0}(x)=G_{0}^{\prime}(x)$, and multivariate odd skewing weight $w(x)$, is defined by $f(x)=2 f_{0}(x) G_{0}\{w(x)\}$. The SSD depends on the skewing distribution and the skewing weight only through the perturbation function $G(x)=G_{0}\{w(x)\}$ such that $G(x) \geq 0$ and the reflective property $G(x)+G(-x)=$ 1 holds. Conversely, any function $G(x)$ that satisfies these conditions ensures that $f(x)=2 f_{0}(x) G(x)$ is a density, which represents the SSD formulation adopted by Wang et al. [2]. In fact, any probability density function admits a uniquely defined SSD representation, as shown first by Wang et al. [2], Proposition 3. Azzalini and Regoli [3] refine this result to the representation of a density with arbitrary support in Proposition 2.

The present note considers the multivariate likelihood ratio order for multivariate skew symmetric distributions with a common kernel. We obtain two general sufficient conditions in terms of a reverse hazard rate order (Theorem 4) and a weak reverse hazard rate order (Theorem 7) between perturbation functions. The second sufficient condition is related to Theorem 6.B.8 in Shaked and Shanthikumar [4], which establishes a sufficient condition for the stochastic order. It is simpler and implies even the likelihood ratio order.

\section{Multivariate Likelihood Ratio Order}

Unless otherwise stated, $X=\left(X_{1}, \ldots, X_{n}\right)$ and $Y=$ $\left(Y_{1}, \ldots, Y_{n}\right)$ denote throughout real random vectors on some probability space with supports $S(X)$ and $S(Y)$. We assume that $X$ and $Y$ have skew-symmetric distributions (SSD) in the sense of Azzalini and Capitanio [1] and Wang et al. [2] as unified in Azzalini and Regoli [3]. Our analysis is restricted to SSDs with a common kernel. This means that there exists a continuous centrally symmetric (about 0 ) density function $f_{0}(x)$, called kernel, and (reflective) perturbation functions $G_{X}(x)$ and $G_{Y}(x)$ satisfying the conditions

$$
\begin{array}{ll}
G_{X}(x) \geq 0, & G_{X}(-x)=\bar{G}_{X}(x), \\
G_{Y}(x) \geq 0, & G_{Y}(-x)=\bar{G}_{Y}(x),
\end{array}
$$

where the notation $\bar{G}(x)=1-G(x)$ is used throughout, such that the probability density functions (pdf) of $X$ and $Y$ are given by

$$
\begin{aligned}
& f_{X}(x)=2 f_{0}(x) G_{X}(x), \quad x \in S(X), \\
& f_{Y}(y)=2 f_{0}(y) G_{Y}(y), \quad y \in S(Y) .
\end{aligned}
$$

Equivalently to (2), there exists a continuous skewing distribution $G_{0}(x)$ with an even density $g_{0}(x)=G_{0}^{\prime}(x)$ and odd skewing weights $w_{X}(x), w_{Y}(x)$ such that

$$
\begin{array}{ll}
f_{X}(x)=2 f_{0}(x) G_{0}\left\{w_{X}(x)\right\}, & x \in S(X), \\
f_{Y}(y)=2 f_{0}(y) G_{0}\left\{w_{Y}(y)\right\}, & y \in S(Y) .
\end{array}
$$


Following Azzalini and Regoli [3, equation (9)], this equivalence is underpinned by the standard choice (made throughout) of a uniform $U(-1 / 2,1 / 2)$ random variable with distribution

$$
\begin{gathered}
G_{0}(t)=\left(t+\frac{1}{2}\right) \cdot 1_{(-1,1)}(2 t)+1_{[1, \infty)}(2 t), \quad t \in R \\
w(x)=G(x)-\frac{1}{2}
\end{gathered}
$$

where $1_{A}(\cdot)$ denotes the indicator function of the set $A$. With this, properties of perturbation functions directly translate into properties of skewing weights and vice versa.

It is instructive to begin with the univariate case.

Proposition 1 (univariate likelihood ratio order characterization). Suppose that $X$ and $Y$ are SSD random variables with a common kernel, absolutely continuous perturbation functions $G_{X}$ and $G_{Y}$ with derivatives $g_{X}(x)=G_{X}^{\prime}(x), g_{Y}(y)=G_{Y}^{\prime}(y)$. The following conditions are equivalent:

(LR1) $X \leq_{l r} Y$ (X precedes $Y$ in likelihood ratio order).

(LR2) The ratio of the perturbation functions $G_{Y}(t) / G_{X}(t)$ is monotone increasing in $t$ over the union of the supports $S(X) \cup S(Y)$.

(LR3) The reverse hazard rates of the perturbation functions satisfy the inequalities

$$
r_{X}(t)=\frac{g_{X}(t)}{G_{X}(t)} \leq r_{Y}(t)=\frac{g_{Y}(t)}{G_{Y}(t)}, \quad t \in S(X) \cup S(Y) \text {. }
$$

Proof. By definition $X \leq_{\operatorname{lr}} Y$ if, and only if (e.g. Shaked and Shanthikumar [4, equation (1.C.2)]), one has

$$
f_{X}(x) f_{Y}(y) \geq f_{X}(y) f_{Y}(x), \quad \forall x \leq y,
$$

or equivalently, for a SSD with a common kernel,

$$
\frac{G_{Y}(x)}{G_{X}(x)} \leq \frac{G_{Y}(y)}{G_{X}(y)}
$$

that is, (LR2). On the other hand, in case $G_{Y}(t) / G_{X}(t)$ is monotone increasing, so is its logarithmic. The equivalence of (LR2) and (LR3) follows from the relation

$$
\frac{d}{d t} \ln \left\{\frac{G_{Y}(t)}{G_{X}(t)}\right\}=\frac{g_{Y}(t)}{G_{Y}(t)}-\frac{g_{X}(t)}{G_{X}(t)}=r_{Y}(t)-r_{X}(t) .
$$

Though perturbation functions are more general than distribution functions, it is convenient to order them in a similar fashion. With this convention in mind, the univariate likelihood ratio order $X \leq_{\mathrm{lr}} Y$ between two SSD's with a common kernel is equivalent to the reverse hazard rate order $G_{X} \leq_{\mathrm{rh}} G_{Y}$ between its perturbation functions. This follows from Proposition 1 by noting that (LR2) and (LR3) identify with the conditions (1.B.40) and (1.B.42) in Shaked and Shanthikumar [4] for the reverse hazard rate order.

In the multivariate case, the stated unaltered characterization of the likelihood ratio order does not hold. However, when the kernel density $f_{0}$ is multivariate totally positive of order $2\left(\mathrm{MTP}_{2}\right.$ property) and $G_{X} \leq_{\mathrm{rh}} G_{Y}$ in the multivariate reverse hazard rate order, then the likelihood ratio order is fulfilled, as shown in Theorem 4 .

Definition 2. The random vector $X$ is said to be smaller than $Y$ in the likelihood ratio order or $\mathrm{TP}_{2}$ order, written as $X \leq_{\operatorname{lr}} Y$ or $X \leq_{\mathrm{TP}_{2}} Y$, if

$$
f_{X}(x) f_{Y}(y) \leq f_{X}(x \wedge y) f_{Y}(x \vee y), \quad \forall x, y \in R^{n}
$$

where one sets $x \wedge y=\left(x_{1} \wedge y_{1}, \ldots, x_{n} \wedge y_{n}\right), x \vee y=\left(x_{1} \vee\right.$ $\left.y_{1}, \ldots, x_{n} \vee y_{n}\right)$, and $x_{i} \wedge y_{i}=\min \left(x_{i}, y_{i}\right), x_{i} \vee y_{i}=\max \left(x_{i}, y_{i}\right)$. It is important to remark that the multivariate order $\leq_{\mathrm{lr}}$ (as well as the reverse hazard rate order introduced later) is not an order in the usual sense because it does not satisfy the reflexive property (e.g. Shaked and Shanthikumar [4], p.291 and 298). In fact, $X \leq_{1 \mathrm{r}} X$ means that the density $f_{X}(x)$ is multivariate totally positive of order $2\left(\mathrm{MTP}_{2}\right)$ such that

$$
f_{X}(x) f_{X}(y) \leq f_{X}(x \wedge y) f_{X}(x \vee y), \quad \forall x, y \in R^{n},
$$

a property discussed in Karlin and Rinott [5] and Whitt [6].

Applied to SSD random vectors $X$ and $Y$ with a common kernel $f_{0}$ and perturbation functions $G_{X}$ and $G_{Y}$, condition (9) means that

$$
\begin{array}{r}
f_{0}(x) f_{0}(y) G_{X}(x) G_{Y}(y) \\
\leq f_{0}(x \wedge y) f_{0}(x \vee y) G_{X}(x \wedge y) G_{Y}(x \vee y), \\
\forall x, y \in R^{n} .
\end{array}
$$

Now, if the random vector $X_{0}$ associated with the kernel density $f_{0}$ satisfies the reflexive property $X_{0} \leq_{\operatorname{lr}} X_{0}$, or equivalently $f_{0}$ is $\mathrm{MTP}_{2}$, and the following inequalities

$$
G_{X}(x) G_{Y}(y) \leq G_{X}(x \wedge y) G_{Y}(x \vee y), \quad \forall x, y \in R^{n},
$$

hold, then clearly (11) is satisfied. But (12) can be used to define a multivariate reverse hazard rate order (for perturbation functions) by paraphrasing the definition of the hazard rate order in Shaked and Shanthikumar [4], Section 6.D, as done for the corresponding univariate orders (Sections 1.B.1 and 1.B.6).

Definition 3. The perturbation function $G_{X}$ is said to be smaller than $G_{Y}$ in the reverse hazard rate order, written as $G_{X} \leq_{\mathrm{rh}} G_{Y}$, if the property (12) is satisfied.

The above discussion leads to the following sufficient condition.

Theorem 4 (first sufficient condition for likelihood ratio order). Let $X$ and $Y$ be SSD random vectors with a common kernel $f_{0}$ and perturbation functions $G_{X}$ and $G_{Y}$. If $f_{0}$ is $M T P_{2}$ and $G_{X} \leq_{r h} G_{Y}$, then $X \leq_{l r} Y$. 
A strong incentive for the use of the likelihood ratio order, which in many situations is easy to verify, is its automatic implication of the multivariate stochastic order (Shaked and Shanthikumar [4], Theorem 6.E.8). Another possible generalization of the univariate likelihood ratio order is to require instead of (9) the condition

$$
f_{X}(y) f_{Y}(x) \leq f_{X}(x) f_{Y}(y), \quad \forall x \leq y .
$$

Here $x \leq y$ denotes the usual componentwise partial order between vectors, which is defined as follows. If $x=$ $\left(x_{1}, \ldots, x_{n}\right)$ and $y=\left(y_{1}, \ldots, y_{n}\right)$ are two n-dimensional vectors, then $x \leq y$ if $x_{i} \leq y_{i}$ for $i=1, \ldots, n$. It is well known that the simpler condition (13) does not necessarily imply the multivariate stochastic order $X \leq_{\text {st }} Y$ (for counterexamples consult Lehmann [7] and Whitt [6]). However, if besides monotonicity of the ratio $f_{Y}(x) / f_{X}(x)$ one requires that the random vector $X$ is associated, then $X \leq_{\text {st }} Y$ (Shaked and Shanthikumar [4], Theorem 6.B.8, p.270). Recall that a random vector $X$ is positively associated if $\operatorname{Cov}[f(X), g(X)] \geq$ 0 for all increasing functions $f, g$ for which the covariance exists. Similarly, the random vector is negatively associated if $\operatorname{Cov}\left[f\left(X_{i}, i \in A\right), g\left(X_{j}, j \in B\right)\right] \leq 0$ for every pair of disjoint subsets $A, B$ of $\{1, \ldots, n\}$, and for all coordinatewise increasing (or decreasing) functions $f, g$ for which the covariance exists. Positive association has been studied by Esary et al. [8] and negative association by Alam and Saxena [9] and Joag-Dev and Proschan [10] (see also Gerasimov et al. [11] for a recent contribution). Now, for SSD random vectors $X$ and $Y$ with a common kernel, it is possible to establish $X \leq_{\text {st }} Y$ from (13) under an alternative simpler assumption to the association of $X$, which even implies likelihood ratio order (Theorem 7).

Condition (13) for SSDs with a common kernel means that

$$
G_{X}(y) G_{Y}(x) \leq G_{X}(x) G_{Y}(y), \quad \forall x \leq y .
$$

Similarly to Shaked and Shanthikumar [4], Section 6.D, and the discussion preceding Definition 3, condition (14) motivates the following ordering between perturbation functions.

Definition 5. The perturbation function $G_{X}$ is said to be smaller than $G_{Y}$ in the weak reverse hazard rate order, written as $G_{X} \leq_{\text {wrh }} G_{Y}$ if the property (14) is satisfied.

From (12) and (14), it follows immediately that

$$
G_{X} \leq_{\mathrm{rh}} G_{Y} \Longrightarrow G_{X} \leq_{\text {wrh }} G_{Y} .
$$

To establish a converse to (15), a result similar to Theorem 6.D.1 in Shaked and Shanthikumar [4] is required.

Lemma 6. Assume that the perturbation functions $G_{X}$ and $G_{Y}$ have a common support $S$, which is a lattice (that is, if $x, y \in S$ then $x \wedge y, x \vee y \in S$ ). If $G_{X}$ and/or $G_{Y}$ is $M_{T}$, then

$$
G_{X} \leq_{w r h} G_{Y} \Longrightarrow G_{X} \leq_{r h} G_{Y} .
$$

Proof. The ordering $G_{X} \leq_{\text {wrh }} G_{Y}$ implies by (14) that

$$
G_{X}(x \vee y) G_{Y}(y) \leq G_{X}(y) G_{Y}(x \vee y), \quad \forall x, y \in R^{n} .
$$

If $G_{X}$ is $\mathrm{MTP}_{2}$, then by the defining property (10) one has

$$
G_{X}(x) G_{X}(y) \leq G_{X}(x \wedge y) G_{X}(x \vee y), \quad \forall x, y \in R^{n} .
$$

Multiplication of these two inequalities yields

$$
\begin{array}{r}
G_{X}(x \vee y) G_{Y}(y) G_{X}(x) G_{X}(y) \\
\leq G_{X}(y) G_{Y}(x \vee y) G_{X}(x \wedge y) G_{X}(x \vee y), \\
\forall x, y \in R^{n} .
\end{array}
$$

Now, since $S$ is a lattice, it follows that if $G_{X}(x) G_{X}(y)>0$, then $G_{X}(y)$ and $G_{X}(x \vee y)$ are positive. Cancelling these quantities in the preceding inequality implies that (12) holds in this case. If $G_{X}(x) G_{X}(y)=0$ then (12) is trivially fulfilled. Together, this shows that $G_{X} \leq_{\mathrm{rh}} G_{Y}$. If $G_{Y}$ is $\mathrm{MTP}_{2}$, one shows similarly that $G_{X} \leq_{\mathrm{rh}} G_{Y}$. The proof is complete.

Theorem 7 (second sufficient condition for likelihood ratio order). Let $X$ and $Y$ be SSD random vectors with a common kernel $f_{0}$ and perturbation functions $G_{X}$ and $G_{Y}$, which have a common lattice support. If $f_{0}$ is $M T P_{2}$, at least one of $G_{X}$ and $G_{Y}$ is $M T P_{2}$, and $G_{X} \leq_{w r h} G_{Y}$; then $X \leq_{l r} Y$.

Proof. The result follows from Lemma 6 and Theorem 4 .

As a consequence, we derive two perturbation invariant stochastic order results for the SSD class. Recall that under perturbation invariance one understands general statements about the random vectors $X_{0}$ and $X$ associated with the kernel densities $f_{0}(x)$ and the SSD densities $f_{X}(x)=$ $2 f_{0}(x) G_{X}(x)$ that remain valid over a large class of perturbation functions. For example, the well-known even transformation invariance states that

$$
T(X)={ }_{d} T\left(X_{0}\right) \text { (equality in distribution), }
$$

for all even real functions $T(x)$, whatever the perturbation function $G_{X}(x)$ is (e.g., Azzalini and Regoli [3], Proposition 1).

Corollary 8 (likelihood ratio order invariance). Let $f_{X}(x)=$ $2 f_{0}(x) G_{X}(x)$ be the density function of the SSD random vector $X$, and let $X_{0}$ be the random vector associated with the kernel density $f_{0}$. Assume that $f_{0}$ is $M T P_{2}$ and the support of $S(X)$ is a lattice. Then, the following likelihood ratio order invariant properties hold:

(LRI1) if $G_{X}$ is an increasing perturbation function then $X_{0} \leq_{l r} X$

(LRI2) if $G_{X}$ is decreasing on $S(X) \cap R_{+}^{n}$, then $X \leq_{l r} X_{0}^{+}=X_{0}$. $1_{R_{+}^{n}}\left(X_{0}\right)$.

Proof. To show (LRI1) it suffices to observe that the perturbation function $G_{X_{0}}(x) \equiv 1 / 2$ is $\mathrm{MTP}_{2}$ and generates the kernel density $f_{0}(x)$ of $X_{0}$. The statement follows from Theorem 7. Similarly, the choice $G_{X_{0}^{+}}(x)=1_{R_{+}^{n}}(x)$ generates the random variable $X_{0}^{+}$and (LRI2) follows also from Theorem 7. 


\section{Discussion and Conclusions}

It appears useful to discuss what has been obtained. So far, in the already large literature on skew-symmetric distributions, only a limited number of results establish in advance formal properties of the SSD by given qualitative properties of the kernel, skewing distribution and skewing weight, or equivalently by given kernel and perturbation function. The even transformation invariance property (20) is the most prominent result of this kind. Azzalini and Regoli [3], Proposition 3, derive the following remarkable new characterization result. If even transformation invariance holds between random vectors $X$ and $Y$ for all even $T(x)$ that is, $T(X)={ }_{d} T(Y)$, then the corresponding densities admit necessarily a representation with a common kernel. In the present note, we have studied a bit further the latter class of SSDs with a common kernel. We have derived two general sufficient conditions for the multivariate likelihood ratio order in Theorems 4 and 7. Since the validity of such an ordering relationship automatically implies the multivariate stochastic order, it has potential for applications in statistics. Also, a novel likelihood ratio order perturbation invariance property has been displayed in Corollary 8. Finally, we remark that the sufficient conditions $G_{X} \leq_{\text {wrh }} G_{Y}$ in Theorem 7 and $G_{X} \leq_{\mathrm{rh}} G_{Y}$ in Theorem 4 can be viewed as multivariate extensions of the criteria (LR2) and (LR3) in Proposition 1. It can be asked whether (in generalization to the univariate case) converses to these results also hold.

\section{References}

[1] A. Azzalini and A. Capitanio, "Distributions generated by perturbation of symmetry with emphasis on a multivariate skew $t$-distribution," Journal of the Royal Statistical Society B, vol. 65 , no. 2, pp. 367-389, 2003.

[2] J. Wang, J. Boyer, and M. G. Genton, "A skew-symmetric representation of multivariate distributions," Statistica Sinica, vol. 14, no. 4, pp. 1259-1270, 2004.

[3] A. Azzalini and G. Regoli, "Some properties of skew-symmetric distributions," Annals of the Institute of Statistical Mathematics, vol. 64, no. 4, pp. 857-879, 2012.

[4] M. Shaked and J. G. Shanthikumar, Stochastic Orders, Springer, New York, NY, USA, 2007.

[5] S. Karlin and Y. Rinott, "Classes of orderings of measures and related correlation inequalities. I. Multivariate totally positive distributions," Journal of Multivariate Analysis, vol. 10, no. 4, pp. 467-498, 1980.

[6] W. Whitt, "Multivariate monotone likelihood ratio and uniform conditional stochastic order," Journal of Applied Probability, vol. 19, no. 3, pp. 695-701, 1982.

[7] E. L. Lehmann, “Ordered families of distributions," Annals of Mathematical Statistics, vol. 26, pp. 399-419, 1955.

[8] J. D. Esary, F. Proschan, and D. W. Walkup, "Association of random variables, with applications," Annals of Mathematical Statistics, vol. 38, pp. 1466-1474, 1967.

[9] K. Alam and K. M. L. Saxena, "Positive dependence in multivariate distributions," Communications in Statistics, vol. 10, no. 12, pp. 1183-1196, 1981.
[10] K. Joag-Dev and F. Proschan, "Negative association of random variables, with applications," The Annals of Statistics, vol. 11, no. 1, pp. 286-295, 1983.

[11] M. Gerasimov, V. Kruglov, and A. Volodin, "On negatively associated random variables," Lobachevskii Journal of Mathematics, vol. 33, no. 1, pp. 47-55, 2012. 


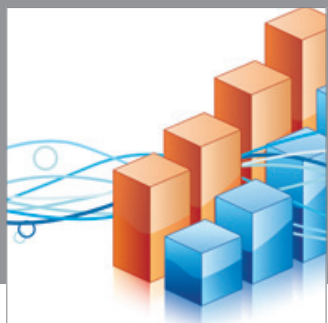

Advances in

Operations Research

mansans

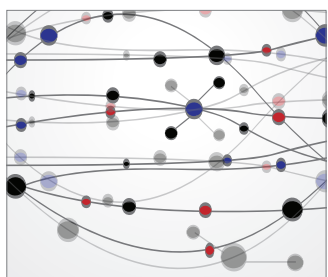

The Scientific World Journal
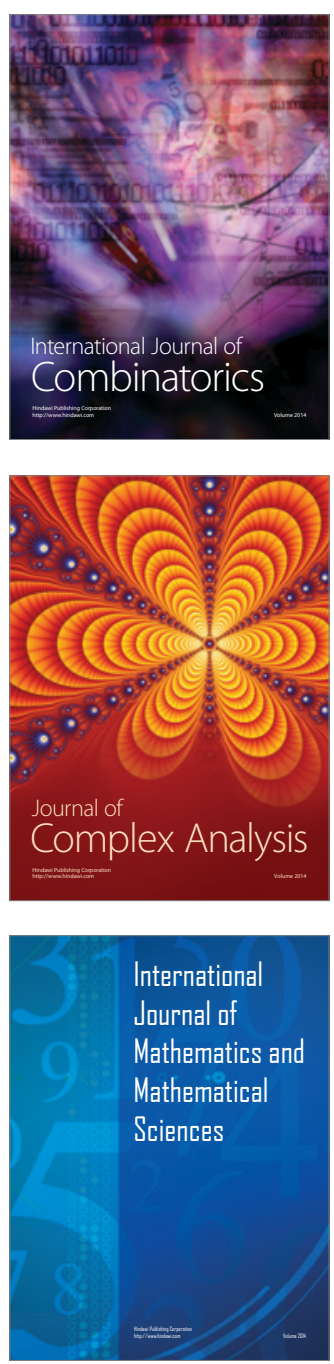
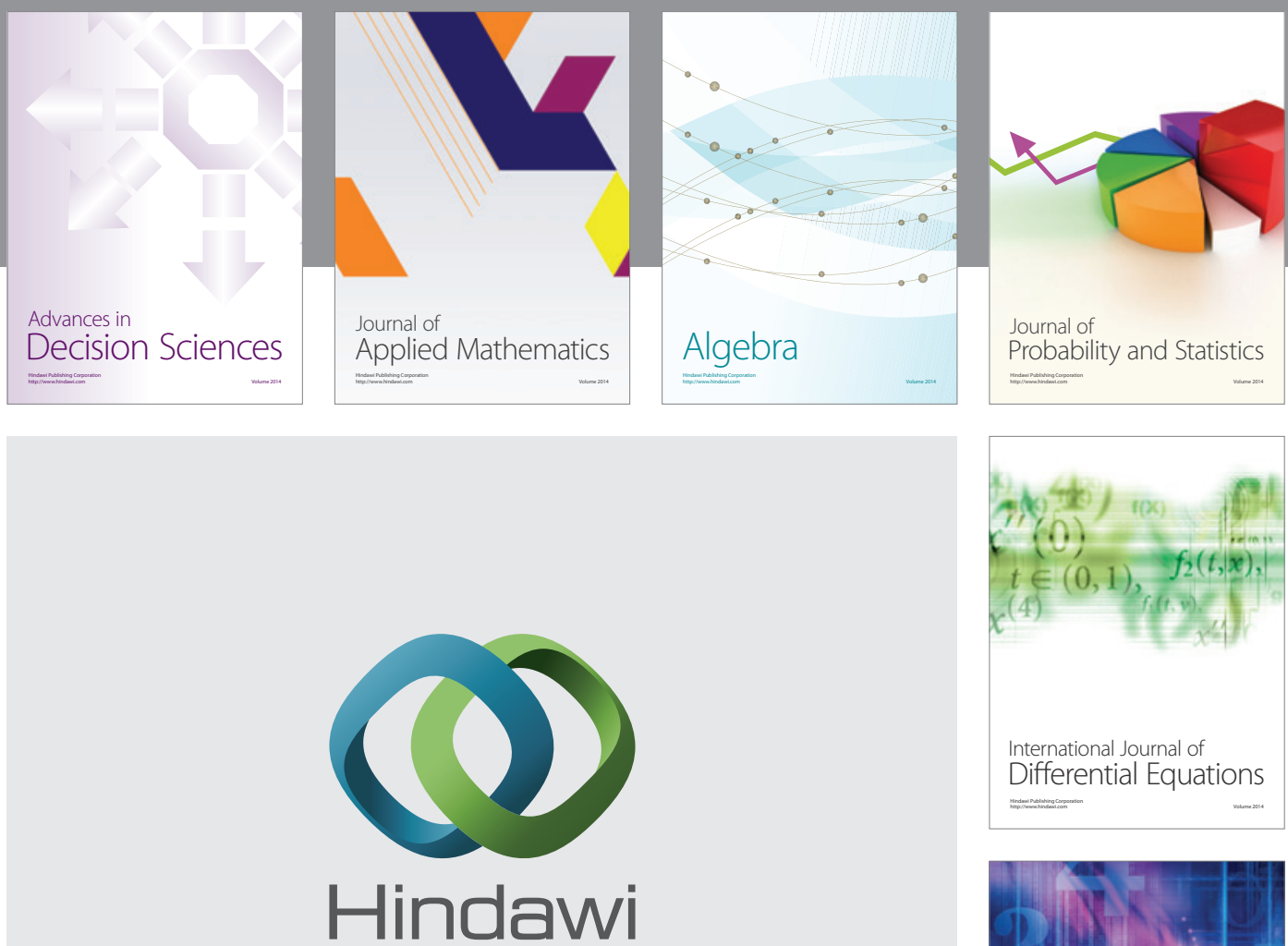

Submit your manuscripts at http://www.hindawi.com
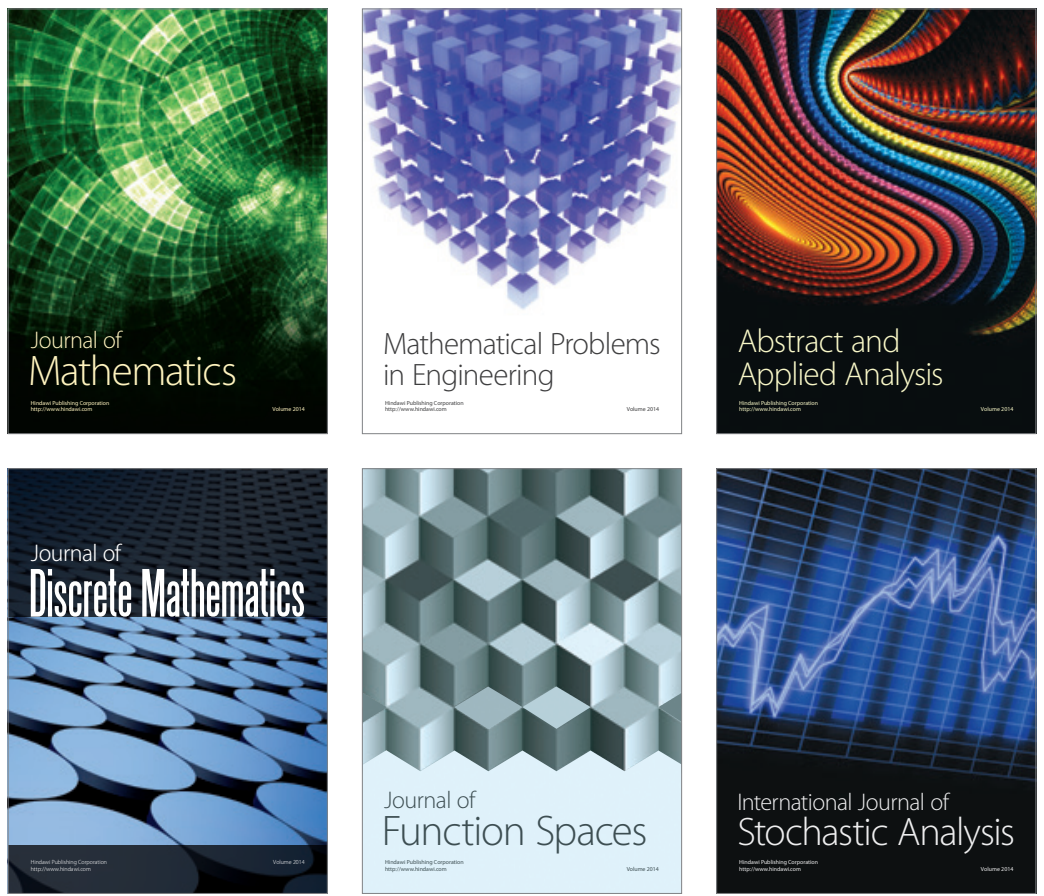

Journal of

Function Spaces

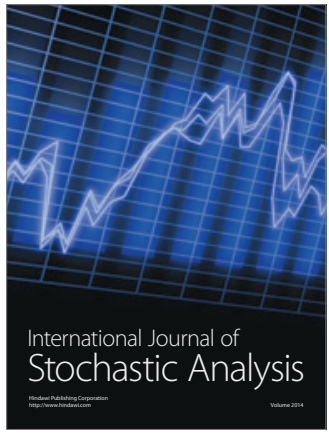

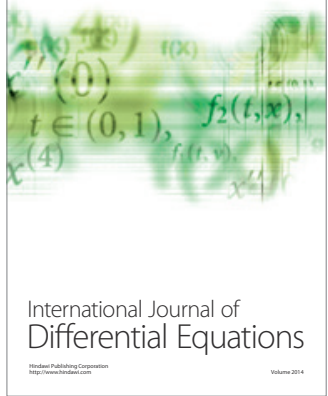
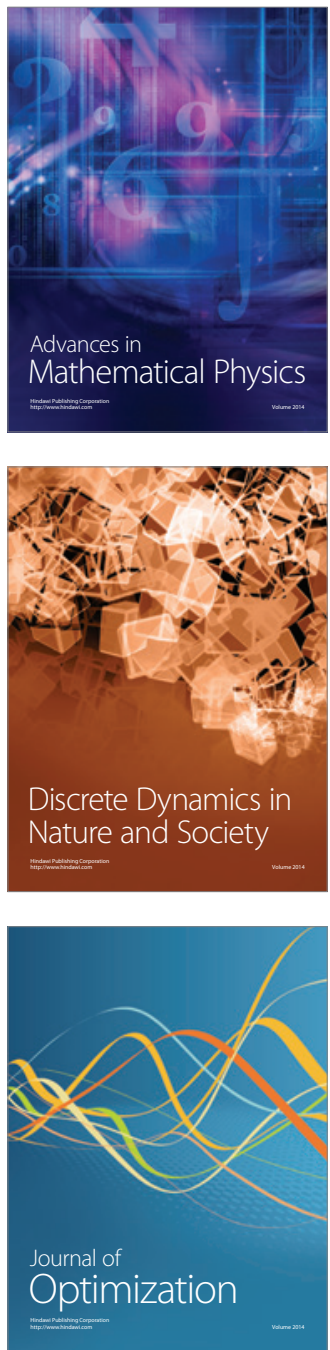